\title{
Rheological characterization of the 2018 Kilauea LERZ Eruptive Sequence
}

\author{
SOldati A. 1 , DingWELl D.B. 1 , \\ HOUGHTON B.2, WALKER B. 2
}

1Department für Geo- und Umweltwissenschaften, LMU München, Theresienstr. 41, 80333 München; arianna.soldati@min.uni-muenchen.de; dingwell@lmu.de 2Department of Earth Sciences, University of Hawai'i at Mānoa, 1680 East-West Road, Honolulu, HI 96822; bhought@soest.hawaii.edu; bhw@hawaii.edu

Magma rheology influences both the dynamic transport of stored magma from subvolcanic depths to the surface and the surface expression of its flow until termination by final cooling and/or topography. Behind all of this is a temperature- chemistry- and crystallinity-dependent rheology which can be estimated in the field and highly precisely determined under controlled conditions in the laboratory. Here, we have determined the rheology of both fully molten and partially crystallized eruption products of the 2018 LERZ eruption. Samples span the entire eruptive sequence, from Early Phase I to Phase III, including the offset Fissure 17. Experiments on fully molten samples were conducted on samples of nine vents using concentric-cylinder rheometry in air in the $1500-1200^{\circ} \mathrm{C}$ temperature range (superliquidus). The liquid viscosities range from $2 \mathrm{~Pa}$ s to $60 \mathrm{~Pa}$ s for Phase I-Phase III samples, and from $5 \mathrm{~Pa}$ s to $917 \mathrm{~Pa}$ s for Fissure 17 samples. To quantify the influence of crystallisation on the viscosity these samples, experiments conducted in air are limited in their applicability (due to the $f \mathrm{O}_{2}$ dependence of liquidus solidus relations). For this reason, we performed a series of viscosity determinations under reducing conditions $\left(f \mathrm{O}_{2}=-7\right)$ in order to stabilise and reproduce the crystallisation sequence observed in nature. Initial crystallisation experiments under equilibrium conditions (isothermal) provide a basis for the interpretation of rheological data as a function of crystal fraction and aspect ratio, as obtained from SEM images of quenched products. Finally, in order to approach most closely the highly dynamic natural conditions of the eruption, we also conducted experiments under disequilibrium crystallisation conditions (under constant cooling rate). We observe that the temperature at which magma stops flowing depends on both cooling rate (primary) and strain rate (secondary). We synthesise our findings in an empirical equation. These data represent the most extensive rheological characterisation of a basaltic eruption sequence to date. 